\title{
EQUAÇÕES PARA ESTIMAR A QUANTIDADE DE CARBONO NA PARTE AÉREA DE ÁRVORES DE EUCALIPTO EM VIÇOSA, MINAS GERAIS ${ }^{1}$
}

\author{
Carlos Pedro Boechat Soares ${ }^{2}$ e Marcio Leles Romarco de Oliveira ${ }^{3}$
}

\begin{abstract}
RESUMO - Este trabalho foi realizado com o objetivo de ajustar equações que permitem estimar com precisão a quantidade de carbono presente na parte aérea das árvores de eucalipto, na região de Viçosa, Minas Gerais. Após as análises constatou-se que: a) o fuste é o componente da parte aérea da árvore com a maior quantidade de carbono estocado, seguido dos galhos, da casca e das folhas; b) existe uma relação exponencial positiva entre a quantidade de carbono presente nas diferentes partes das árvores $\left(Y_{i}\right)$ e as dimensões dos fustes das árvores, expressa pelo dap e altura total $(H t)$, que pode ser descrita pela seguinte relação alométrica: $Y_{i}=a . d a p^{b}$. $H t^{c}$; e c) a variável dap foi significativa, a $95 \%$ de probabilidade, nas equações que estimam a quantidade de carbono de todos os componentes da parte aérea das árvores de eucalipto. Contudo, a variável altura foi significativa apenas nas equações para o fuste e para os galhos.
\end{abstract}

Palavras-chave: Seqüestro de carbono, eucalipto e equações.

\section{EQUATIONS FOR ESTIMATING THE AMOUNT OF CARBON IN THE AERIAL PARTS OF EUCALYPT TREES IN VIÇOSA, MG, BRAZIL}

\begin{abstract}
The objective of this work was to adjust equations that allow for an accurate estimation of the amount of carbon present in the aerial part of eucalypt trees in Viçosa, Minas Gerais, Brazil. After the analyses, it was verified that: a) the stem in the aerial part component of the trees with the largest amount of stored carbon, followed by the branches, bark and leaves; b) there is a positive exponential relationship among the amounts of the carbon present in the different parts of the trees $(\boldsymbol{Y})$ and the stem dimensions of the trees, expressed by diameter at breast height (dbh) and total height $(\boldsymbol{H t})$, that can be described by the following allometric relationship:

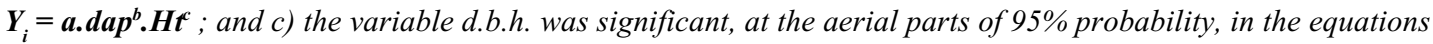
estimating the amount of carbon of all the components of the trees, the variable height was significant only for the stem and branch equations.
\end{abstract}

Key words: Carbon sequestration, eucalypt and equations.

\section{INTRODUÇÃO}

Anualmente, uma grande quantidade de carbono vindo da queima de combustíveis fósseis e de mudanças do uso da terra é lançada à atmosfera. Como conseqüência, há o aumento da concentração de gás carbônico $\left(\mathrm{CO}_{2}\right)$, causando o chamado efeito estufa.
De acordo com especialistas de todo o mundo, esse aumento da concentração de gás carbônico pode resultar em mudanças permanentes no clima, imprimindo novos padrões no regime dos ventos, na pluviosidade e na circulação dos oceanos, acarretando profundas modificações nas condições de vida na Terra. Assim sendo, tem-se um consenso mundial de que estratégias devem ser

1 Recebido para publicação em 01.10.2001.

Aceito para publicação em 6.11.2002.

2 Prof. do Departamento de Engenharia Florestal da Universidade Federal de Viçosa - DEF/UFV, <csoares@mail.ufv.br>; ${ }^{3}$ Estudante do curso de Pós-Graduação em Ciência Florestal da UFV, <mlromarco@bol.com.br>. 
estudadas e empregadas para redução da concentração do $\mathrm{CO}_{2}$ atmosférico, na tentativa de reduzir o risco de eventuais catástrofes mundiais.

Entre as estratégias para redução da concentração de gás carbônico na atmosfera, destacam-se: a) a redução das emissões por queima de combustíveis fósseis; b) a redução da queima de material vegetal; e c) o seqüestro do carbono através do plantio e manejo de florestas. Considerando que a redução da queima de combustíveis fósseis implica desaquecimento da economia de países desenvolvidos e que as mudanças no uso da terra, principalmente devido aos desmatamentos, dificilmente deixarão de ocorrer, tendo em vista o crescimento da população mundial e a necessidade de abertura de novas áreas para atividades agrícolas e de pecuária, o seqüestro de carbono por florestas torna-se uma atividade econômica atrativa, desde que remunerada adequadamente, e ambientalmente correta, do ponto de vista do seqüestro do carbono atmosférico.

No entanto, existe a necessidade de conhecer os estoques de carbono presentes em diferentes compartimentos da floresta (parte aérea das árvores, sistema radicular, sub-bosque, solo, entre outros) para se conhecer o potencial das florestas no seqüestro de carbono, bem como a sua viabilidade econômica. Trabalhos têm sido realizados, utilizando-se estimativas de volume e biomassa em diferentes partes das árvores e em compartimentos das florestas, as quais são convertidas em quantidades de carbono pela utilização de fatores de conversão, ou seja, a quantidade de carbono estocada nas florestas normalmente é obtida de forma indireta (Cooper, 1983; Brown \& Lugo, 1984; Brown et al.,1986; Schroeder, 1992), principalmente devido ao custo elevado para obtenção da quantidade de carbono presente em diferentes compartimentos da floresta.

Assim sendo, torna-se necessário desenvolver ou utilizar metodologias que possibilitem obter estimativas da quantidade de carbono em diferentes partes das árvores e, conseqüentemente, em diferentes compartimentos da floresta. Diante do exposto e considerando o gênero Eucalyptus como importante para o seqüestro de carbono atmosférico, haja vista o seu rápido crescimento e a sua alta produtividade, o objetivo deste trabalho foi ajustar equações que permitem estimar com precisão a quantidade de carbono presente na parte aérea das árvores de eucalipto.

R. Árvore, Viçosa-MG, v.26, n.5, p.533-539, 2002

\section{MATERIAL E MÉTODOS}

O presente trabalho foi realizado com dados de um povoamento de Eucalyptus grandis W. Hill ex-Maiden com 77 meses de idade, localizado no município de Viçosa, Estado de Minas Gerais, Brasil.

Após a caracterização da estrutura diamétrica do povoamento foram selecionadas 40 árvores, nas quais foram medidos o diâmetro com casca do fuste a $1,30 \mathrm{~m}$ (dap) e a altura total das árvores ( $\mathrm{Ht}$ ), bem como realizouse a determinação do volume do fuste com e sem casca, pela aplicação sucessiva da expressão de Smalian (Husch et al., 1993), até um diâmetro-limite de $3 \mathrm{~cm}$.

Cada árvore-amostra foi desfolhada, sendo suas folhas pesadas e ensacadas. Do conjunto de folhas destacadas retirou-se uma amostra de aproximadamente $50 \mathrm{~g}$, para determinação do seu peso seco, após secagem em estufa de circulação forçada de ar a $75 \pm 2$ oC.

Em seguida, cada árvore-amostra foi desgalhada e todos os seus galhos foram pesados, inclusive a ponta da árvore com diâmetro menor que $3 \mathrm{~cm}$. No conjunto de galhos foram retirados fragmentos de galhos localizados em diferentes posições da copa da árvore, os quais foram misturados, formando-se uma amostra composta. Desta amostra, retirou-se uma subamostra de aproximadamente $50 \mathrm{~g}$, para determinação do seu peso seco, após secagem em estufa de circulação forçada de ar a $103 \pm 2{ }^{\circ} \mathrm{C}$.

O tronco de cada árvore-amostra, após as medições para cubagem rigorosa, foi seccionado para extração de discos de madeira com casca de aproximadamente $2,5 \mathrm{~cm}$ de espessura, nos seguintes níveis de medição: $0,25,50$, 75 e $100 \%$ da altura comercial. Os discos serviram como amostras de madeira e de casca para o cálculo da densidade básica da madeira do fuste $(D B M t)$ e da densidade básica da casca $(D B C)$ de cada árvore-amostra (Vital, 1984).

A biomassa do fuste das árvores-amostra, sem casca, e a biomassa da casca foram obtidas de acordo com a seguinte expressão (Finke Herrera, 1989):

$$
P S(c)=V \cdot(D B M t \text { ou } D B C)
$$

em que $P S(c)=$ biomassa do fuste, sem casca, ou da casca, em kg; $V=$ volume do fuste, sem casca, ou da casca, em $\mathrm{m}^{3} ; D B M t=$ densidade básica média da madeira do fuste, em $\mathrm{kg} / \mathrm{m}^{3}$; e $D B C=$ densidade básica da casca, em $\mathrm{kg} /$ $\mathrm{m}^{3}$. 
A biomassa total do fuste de cada árvore-amostra foi obtida ao somar à biomassa do fuste sem casca a biomassa da casca.

A biomassa de galhos e de folhas de cada árvoreamostra foi obtida pela seguinte expressão:

$$
P S(c)=\frac{P U(c) \cdot P S(a)}{P U(a)}
$$

em que $P S(c)=$ biomassa de galhos ou de folhas, em $\mathrm{kg}$; $P U(c)=$ peso úmido do conjunto de galhos ou de folhas no campo, em kg; $P S(a)=$ peso seco da amostra de galhos ou de folhas, em kg; e $P U(a)=$ peso úmido da amostra de galhos ou de folhas, em kg.

As estimativas da quantidade de carbono presentes no conjunto de folhas e galhos e no fuste com e sem casca das árvores-amostra foram obtidas ao multiplicar as respectivas estimativas de biomassa pelo fator 0,5 , ou seja, considerou-se que em média $50 \%$ da biomassa é composta por carbono.

De posse das estimativas de carbono presentes nas folhas, nos galhos e no fuste das árvores-amostra, foram elaborados gráficos que relacionam as quantidades de carbono e os tamanhos das árvores (dap), objetivando a determinação de relações funcionais que possibilitem a estimação da quantidade de carbono presente nos diversos componentes da parte aérea das árvores.

Para evitar julgamentos pessoais, foram utilizados os seguintes critérios na avaliação da precisão das equações para estimar a quantidade de carbono nas diferentes partes das árvores amostra: a) coeficiente de variação (CV\%); b) coeficiente de determinação $\left(R^{2}\right)$; e c) análise gráfica de resíduos (Paula Neto, 1977).

\section{RESULTADOS E DISCUSSÃO}

Após analisar as estimativas das quantidades médias de carbono presentes nos fustes (com e sem casca), nos galhos e nas folhas das árvores de eucalipto no final do Quadro 1, constatou-se que o fuste sem casca representa a parte aérea da árvore com maior quantidade de carbono $(83,24 \%)$, seguido dos galhos $(6,87 \%)$, da casca $(6,62 \%)$ e das folhas $(2,48 \%)$. Além disto, verificou-se uma nítida tendência de aumento da quantidade de carbono das diferentes partes das árvores com o tamanho das árvores, o que pode ser mais bem visualizado com a Figura 1 .
Os gráficos de dispersões (Figura 1), indicam uma relação exponencial positiva entre a quantidade de carbono presente nas diferentes partes da árvore $(Y)$ e o tamanho das árvores (dap), que pode ser representada pela seguinte relação alométrica (Vann et al., 1998):

$$
Y=a \cdot d a p^{b}
$$

No entanto, como árvores de mesmo diâmetro (dap) podem ter diferentes quantidades de carbono, por possuírem diferentes alturas e formas diferenciadas dos fustes e estarem em diferentes sítios e estratos da floresta, pode-se incluir a variável altura total da árvore $(\mathrm{Ht})$ na expressão 1 como forma de melhorar o poder de explicação da equação. Desta forma, a expressão 1 fica assim redefinida:

$$
Y=a . \operatorname{dap}^{b} . \mathrm{HT}^{\mathrm{c}}
$$

A expressão 2 pode ser caracterizada como uma variação do modelo volumétrico de Schumacher \& Hall (1933), no qual, em vez de estimar o volume do tronco das árvores individualmente, estima-se a quantidade de carbono presente em diferentes componentes da parte aérea da planta.

Em uma primeira análise, a relação apresentada na expressão 2 seria compatível somente para o tronco das árvores, dada à relação direta entre a quantidade de carbono presente no fuste e as dimensões da árvore. Contudo, pode-se estender esta relação para galhos e folhas, haja vista que quanto maior a copa da árvore (maior massa de galhos e folhas), maior deve ser a dimensão do tronco para sua sustentação.

Considerando a facilidade de ajustar uma equação linear através do método dos mínimos quadrados ordinários, além do fato de a linearização da equação poder corrigir eventuais problemas de heterocedasticidade de variância e de normalidade dos erros, optou-se por ajustar a expressão $2 \mathrm{em}$ sua forma linear, obtendo-se os seguintes ajustes:

Fuste (sem casca):

$$
\begin{gathered}
\operatorname{Ln} Y=-6,966833+1,587810 \cdot \operatorname{Ln} d a p^{*}+2,000404 \cdot \operatorname{Ln} H t^{*} \\
\mathrm{R}^{2}=98,82 \% \quad \operatorname{CV}=16,30 \%
\end{gathered}
$$

Fuste (com casca):

$$
\begin{gathered}
\operatorname{Ln} Y=-6,609865+1,661056 \cdot \operatorname{Ln} d a p^{*}+1,851121 \cdot \operatorname{Ln} H t^{*} \\
\mathrm{R}^{2}=98,90 \% \quad \mathrm{CV}=14,98 \%
\end{gathered}
$$

R. Árvore, Viçosa-MG, v.26, n.5, p.533-539, 2002 
Quadro 1 - Estimativas da quantidade de carbono nos fustes, com e sem casca, nos galhos e nas folhas de árvoresamostras de Eucalyptus grandis

Table 1 - Estimates of the amount of carbon in the stems (with and without bark), branches and leaves of sample-trees of Eucalyptus grandis

\begin{tabular}{|c|c|c|c|c|c|c|c|}
\hline \multirow{2}{*}{$\begin{array}{c}\text { Árvore } \\
\left(\mathbf{N}^{\mathbf{0}}\right)\end{array}$} & \multirow{2}{*}{$\begin{array}{c}\text { Classe de } \\
\text { dap } \\
(\mathrm{cm})\end{array}$} & \multirow{2}{*}{$\begin{array}{l}d a p \\
(\mathrm{~cm})\end{array}$} & \multirow{2}{*}{$\begin{array}{l}\mathrm{Ht} \\
(\mathrm{m})\end{array}$} & \multicolumn{4}{|c|}{ Carbono (kg) } \\
\hline & & & & $\begin{array}{c}\text { Fuste } \\
\text { (sem casca) }\end{array}$ & $\begin{array}{c}\text { Fuste } \\
\text { (com casca) }\end{array}$ & Galhos & Folhas \\
\hline 1 & \multirow{5}{*}{6,25} & 6,0 & 12,6 & 2,69 & 2,88 & 0,97 & 0,16 \\
\hline 2 & & 6,7 & 13,9 & 3,65 & 3,99 & 0,50 & 0,14 \\
\hline 3 & & 6,6 & 11,7 & 2,23 & 2,50 & 0,49 & 0,19 \\
\hline 4 & & 7,0 & 12,8 & 4,39 & 5,10 & 0,47 & 0,20 \\
\hline 5 & & 6,8 & 13,3 & 3,03 & 3,45 & 0,53 & 0,15 \\
\hline \multicolumn{3}{|c|}{ Média/Porcentagem Média } & & $3,20(73,54)$ & $3,59(82,44)$ & $0,59(13,61)$ & $0,17(3,95)$ \\
\hline 1 & \multirow{5}{*}{8,75} & 8,6 & 16,3 & 8,79 & 9,49 & 0,58 & 0,36 \\
\hline 2 & & 9,0 & 16,4 & 7,78 & 8,61 & 0,66 & 0,25 \\
\hline 3 & & 8,4 & 15,9 & 7,55 & 8,13 & 0,81 & 0,23 \\
\hline 4 & & 9,1 & 18,8 & 9,59 & 10,69 & 1,32 & 0,26 \\
\hline 5 & & 9,0 & 14,9 & 6,30 & 7,31 & 0,98 & 0,13 \\
\hline \multicolumn{3}{|c|}{ Média/Porcentagem Média } & & $8,01(80,32)$ & $8,85(88,34)$ & $0,87(8,76)$ & $0,25(2,50)$ \\
\hline 1 & \multirow{5}{*}{11,25} & 11,0 & 20,3 & 20,48 & 22,99 & 0,76 & 0,40 \\
\hline 2 & & 11,1 & 22,8 & 20,49 & 21,91 & 0,65 & 0,39 \\
\hline 3 & & 11,3 & 21,9 & 17,87 & 19,98 & 0,68 & 0,33 \\
\hline 4 & & 11,3 & 23,2 & 24,62 & 25,69 & 0,53 & 0,59 \\
\hline 5 & & 11,0 & 20,6 & 22,17 & 23,73 & 1,19 & 0,93 \\
\hline \multicolumn{3}{|c|}{ Média/Porcentagem Média } & & $21,13(88,20)$ & $22,66(94,6)$ & $0,77(3,19)$ & $0,53(2,21)$ \\
\hline 1 & \multirow{5}{*}{13,75} & 13,9 & 23,9 & 35,52 & 37,51 & 1,33 & 0,71 \\
\hline 2 & & 13,5 & 20,9 & 20,29 & 23,17 & 2,07 & 0,32 \\
\hline 3 & & 13,6 & 22,6 & 31,78 & 34,67 & 2,96 & 1,45 \\
\hline 4 & & 13,5 & 21,9 & 25,10 & 27,83 & 1,57 & 0,56 \\
\hline 5 & & 14,0 & 21,1 & 36,29 & 39,52 & 2,72 & 1,29 \\
\hline \multicolumn{3}{|c|}{ Média/Porcentagem Média } & & $29,80(83,84)$ & $32,54(91,55)$ & $2,13(6,00)$ & $0,87(2,45)$ \\
\hline 1 & \multirow{5}{*}{16,25} & 16,2 & 25,9 & 55,87 & 60,01 & 2,40 & 0,70 \\
\hline 2 & & 16,0 & 25,2 & 42,42 & 47,41 & 2,22 & 0,65 \\
\hline 3 & & 16,3 & 26,4 & 68,13 & 73,63 & 5,77 & 1,87 \\
\hline 4 & & 16,4 & 24,0 & 45,46 & 48,40 & 3,19 & 1,49 \\
\hline 5 & & 16,1 & 26,8 & 57,25 & 63,07 & 2,56 & 1,59 \\
\hline \multicolumn{3}{|c|}{ Média/Porcentagem Média } & & $53,83(85,45)$ & $58,50(92,87)$ & $3,23(5,13)$ & $1,26(2,00)$ \\
\hline 1 & \multirow{5}{*}{18,75} & 18,8 & 27,6 & 83,81 & 90,98 & 6,18 & 2,85 \\
\hline 2 & & 18,4 & 26,5 & 60,49 & 65,64 & 2,12 & 1,04 \\
\hline 3 & & 18,6 & 26,4 & 74,73 & 80,60 & 5,18 & 2,04 \\
\hline 4 & & 19,0 & 27,9 & 75,91 & 80,70 & 4,87 & 2,64 \\
\hline 5 & & 18,0 & 28,2 & 64,47 & 70,40 & 4,41 & 1,35 \\
\hline \multicolumn{3}{|c|}{ Média/Porcentagem Média } & & $71,88(85,36)$ & $77,66(92,23)$ & $4,55(5,41)$ & $1,99(2,36)$ \\
\hline 1 & \multirow{5}{*}{21,25} & 21,2 & 27,1 & 78,14 & 87,31 & 6,65 & 1,33 \\
\hline 2 & & 21,0 & 28,9 & 95,00 & 101,56 & 6,67 & 1,80 \\
\hline 3 & & 21,3 & 27,9 & 115,98 & 121,26 & 7,76 & 1,78 \\
\hline 4 & & 21,0 & 30,4 & 104,77 & 112,47 & 6,81 & 2,11 \\
\hline 5 & & 20,7 & 28,8 & 86,87 & 95,52 & 3,24 & 1,52 \\
\hline \multicolumn{3}{|c|}{ Média/Porcentagem Média } & & $96,16(86,19)$ & $103,62(92,88)$ & $6,23(5,58)$ & $1,71(1,53)$ \\
\hline 1 & \multirow{5}{*}{23,75} & 23,7 & 26,4 & 100,48 & 110,22 & 8,55 & 1,68 \\
\hline 2 & & 23,4 & 29,4 & 137,99 & 147,96 & 13,09 & 5,17 \\
\hline 3 & & 23,6 & 29,5 & 112,95 & 122,89 & 9,34 & 4,12 \\
\hline 4 & & 24,0 & 30,0 & 155,84 & 166,78 & 10,01 & 5,51 \\
\hline 5 & & 23,9 & 28,0 & 98,93 & 108,38 & 11,57 & 4,14 \\
\hline Médi & rcentagem & & & $121,24(83,01)$ & $131,25(89,86)$ & $10,68(7,31)$ & $4,13(2,83)$ \\
\hline Médi & ral em kg e & entage & & $50,65(83,24)$ & $54,83(89,86)$ & $3,63(6,87)$ & $1,36(2,48)$ \\
\hline
\end{tabular}


Galhos:

$$
\begin{gathered}
\operatorname{Ln} Y=-1,695267+3,888792 \cdot \operatorname{Ln} d a p^{*}-2,492777 \cdot \operatorname{Ln} H t^{*} \\
\mathrm{R}^{2}=88,27 \%
\end{gathered}
$$

Folhas:

$$
\begin{gathered}
\operatorname{Ln} Y=-6,649474+2,273838 \cdot \operatorname{Ln} d a p^{*}-0,155153 \cdot \operatorname{Ln} H t^{\text {n.s. }} \\
\mathrm{R}^{2}=85,40 \%
\end{gathered}
$$

Pode-se constatar que o modelo proposto para estimar a quantidade de carbono presente nos fustes, com e sem casca, nos galhos e nas folhas expressou um alto grau de ajuste, principalmente para os fustes da árvore, com e sem casca. Para galhos e folhas houve decréscimo da precisão.

A variável dap foi significativa a $95 \%$ de probabilidade em todas as equações ajustadas $(*)$, confirmando-se
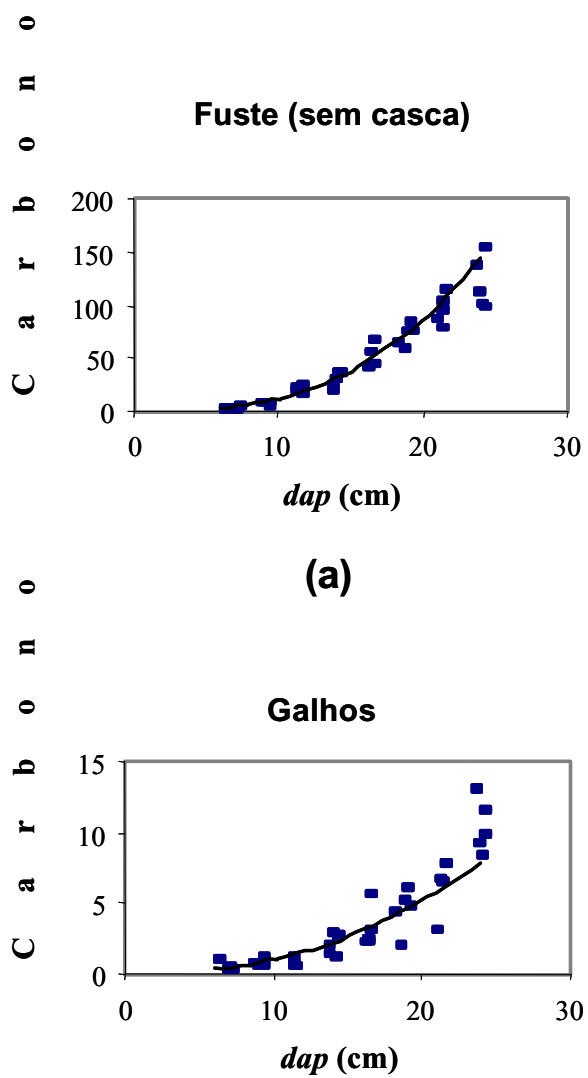

(c) como variável importante nos modelos para estimar a quantidade de carbono da parte aérea das árvores de eucalipto. A variável altura total $(H t)$, por sua vez, foi significativa apenas nas equações que estimam a quantidade de carbono no fuste e nos galhos. A não-significância desta variável na equação para folhas implica que a quantidade de folhas em uma árvore se deve, principalmente, à estação do ano (caducifolismo), à inclinação do terreno e ao tamanho da copa da árvore, e não à altura da árvore propriamente dita. Ou seja, variáveis relacionadas ao tamanho das copas, como altura da copa e diâmetro da copa, devem ser incluídas nos modelos que estimam a quantidade de carbono total de folhas presente em árvores de eucalipto. Satoo e Madgwich, citados por Ferreira (1984), também sugeriram essas variáveis para estimar a biomassa dos componentes de copa das árvores de eucalipto.
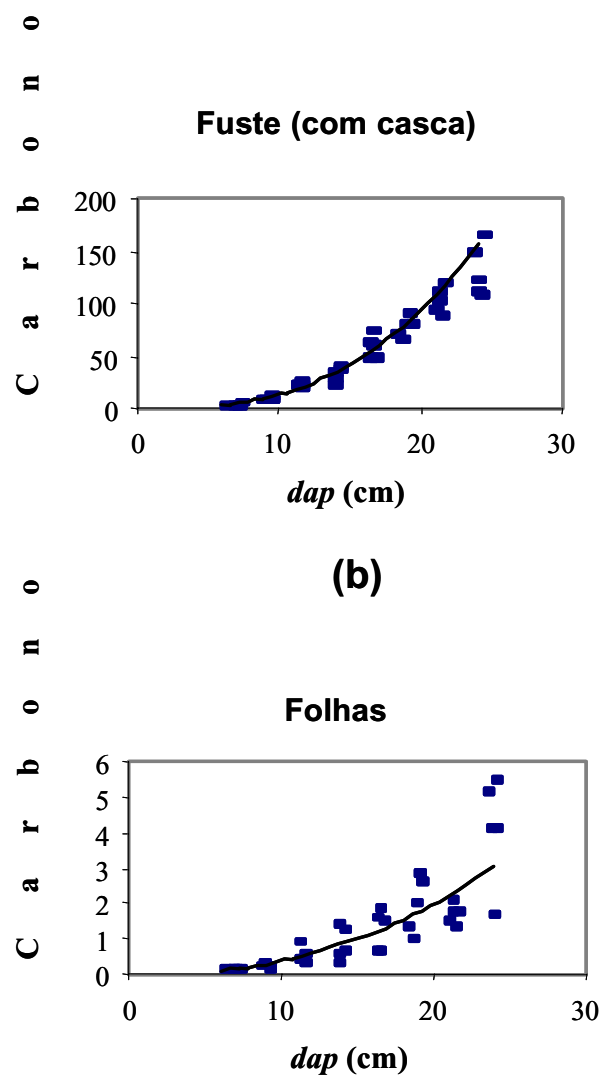

(d)

Figura 1 - Gráficos de dispersão das quantidades de carbono presentes nos fustes sem casca (a), no fuste com casca (b), nos galhos (c) e nas folhas (d), em função dos diâmetros (dap) das árvores-amostra.

Figure 1 -Graphs of dispersion of the amounts of carbon present in the stems without bark (a), stems with bark (b), branches (c) and leaves (d), in function of the diameters at breast height (dbh) of the sample-trees. 


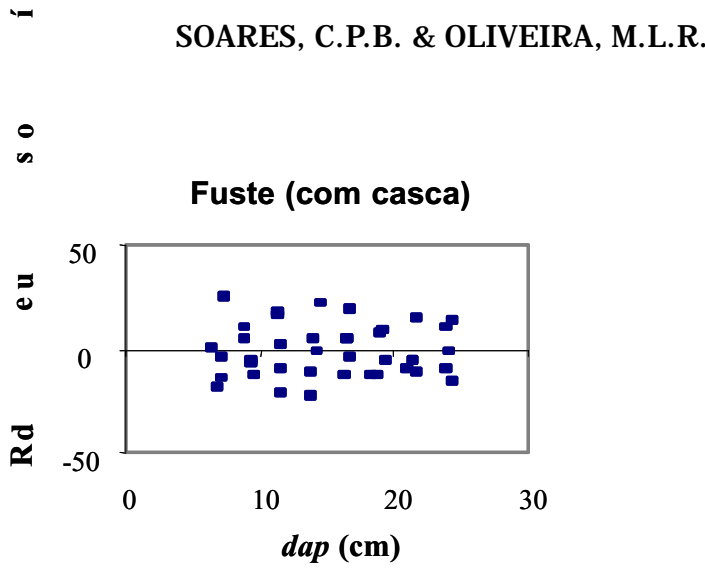

Fuste (sem casca)

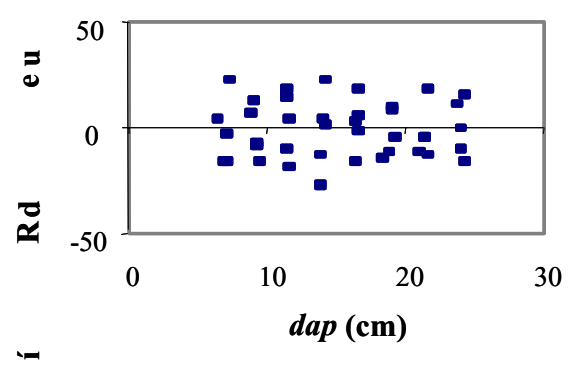

(a)

$n$

Galhos

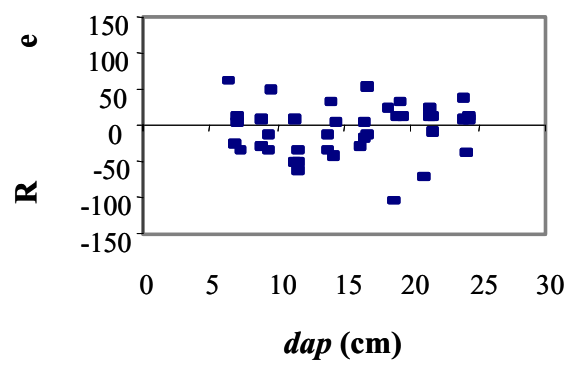

(c) (b)

$\infty$

Folhas

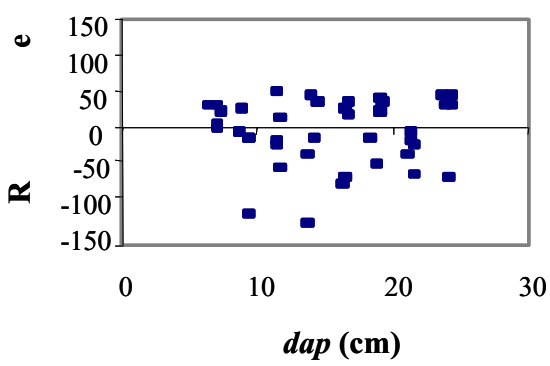

(d)

Figura 2 - Distribuição dos resíduos porcentuais das equações para os fustes sem casca (a), fustes com casca (b), galhos (c) e folhas (d), em função dos diâmetros (dap) das árvores-amostra.

Figure 2 -Distribution of the percentage residues of the equations for stem without bark (a), stem with bark (b), branches (c) and leaves $(d)$, in function of the diameters at breast height (dbh) of the sample-trees.

Analisando os gráficos de resíduos apresentados na Figura 2, observa-se que ocorre uma distribuição dos resíduos mais próximos de zero para o fuste com e sem casca e galhos (Figura 2-A, 2-B e 2-C), definindo um comportamento preciso e homogêneo para todas as classes de diâmetro. Na Figura 2-D ocorre tendência de subestimação da quantidade de carbono para árvores de todos os diâmetros, além de menor precisão, haja vista a amplitude dos resíduos porcentuais.

\section{CONCLUSÕES}

Após as análises constatou-se que: a) o fuste é o componente da parte aérea da árvore com a maior quantidade de carbono estocado, seguido dos galhos, da casca e das folhas; b) existe uma relação exponencial positiva entre a quantidade de carbono presente nas diferentes partes das árvores $\left(Y_{i}\right)$ e as dimensões dos fustes das árvores, expressas pelo dap e pela altura total, que pode ser descrita pela seguinte relação alométrica: $Y_{i}=a \cdot d a p^{b} . H t^{c}$; e c) a variável dap foi significativa, a $95 \%$ de probabilidade, nas equações que estimam a quantidade de carbono de todos os componentes da parte aérea das árvores de eucalipto. Contudo, a variável altura foi significativa apenas nas equações para o fuste e para os galhos.

\section{REFERÊNCIAS BIBLIOGRÁFICAS}

BROWN, S.; LUGO, A. E. Biomass of tropical forest: A new estimate based on forest volumes. Science, v. 223, p. 1290-1293, 1984. 
BROWN, S.; LUGO, A. E.; CHAPMAN, J. Biomass of tropical tree plantations and its implication for the global carbon budget. Canadian Journal of Forest Research, v. 13 , p. $390-394,1986$.

COOPER, C. F. Carbon storage in managed forest.

Canadian Journal of Forest Research, v. 13, n. 1, p. 155$165,1983$.

FERREIRA, M. G. M. An analysis of the productivity of Eucalyptus grandis plantations in the "cerrado" region in Brazil: A nutrient ajeling approach. Vancouver: UBC, 1984. 230 p. Thesis (Ph.D.) - University of British Columbia, 1984.

FINKE HERREIRA, M. C. Densidade básica e equações de peso de madeira seca de povoamentos de eucalipto de acordo com a idade, espécie e método de regeneração. Viçosa: UFV, 1989. 113 p. Dissertação (Mestrado em Ciência Florestal) - Universidade Federal de Viçosa, 1989.
HUCH, B.; MILLER, C. I.; BEERS, T. W. Forest mensuration. 3.ed. Florida: Krieger Publishing company. 1993. $402 \mathrm{p}$.

PAULA NETO, F. Tabelas volumétricas com e sem casca para Eucalyptus saligna. Revista Árvore, v. 1, n. 1, p. 3154,1977

SCHROEDER, P. Carbon storage potencial of short rotation tropical tree plantations. Forest Ecology and Management, v. 50, p. 31-41, 1992.

SCHUMACHER, F. X.; HALL, F. S. Logarithmic expression of timber-tree volume. Journal of Agricultural Research, v. 47, n. 9, p. 719-734, 1933.

VANN, D. R.; PÁLMIOTTO, P. A. ; STRIMBECK, G. R. Allometric equations for two South American conifers: Test of a non-destructive method. Forest Ecology and Management, v. 106, p. 55-71, 1998.

VITAL, B. R. Métodos de determinação da densidade da madeira. Boletim Técnico SIF, n. 1, p. 1-21, 1984. 